\title{
Computer-Assisted Ankle Joint Arthroplasty Using Bio-engineered Autografts
}

\author{
R. Sidler ${ }^{1}$, W. Köstler ${ }^{2}$, T. Bardyn ${ }^{1}$, M.A. Styner ${ }^{3}$, N. Südkamp ${ }^{2}$, \\ L. Nolte ${ }^{1}$, and M. Á. González Ballester ${ }^{1}$ \\ 1 MEM Research Center, Institute for Surgical Technology \\ and Biomechanics, University of Bern, Switzerland \\ rudolf.sidler@MEMcenter.unibe.ch \\ ${ }^{2}$ Universitätsklinikum Freiburg, \\ Dept. of Orthopaedics and Traumatology, Freiburg i.B., Germany \\ 3 Departments of Computer Science and Psychiatry, \\ University of North Carolina at Chapel Hill, U.S.A.
}

\begin{abstract}
Bio-engineered cartilage has made substantial progress over the last years. Preciously few cases, however, are known where patients were actually able to benefit from these developments. In orthopaedic surgery, there are two major obstacles between in-vitro cartilage engineering and its clinical application: successful integration of an autologuous graft into a joint and the high cost of individually manufactured implants. Computer Assisted Surgery techniques can potentially address both issues at once by simplifying the therapy, allowing pre-fabrication of bone grafts according to a shape model, individual operation planning based on CT images and providing optimal accuracy during the intervention. A pilot study was conducted for the ankle joint, comprising a simplified rotational symmetric bone surface model, a dedicated planning software and a complete cycle of treatment on one cadaveric human foot. The outcome was analysed using CT and MRI images; the post-operative CT was further segmented and registered with the implant shape to prove the feasibility of computer assisted arthroplasty using bio-engineered autografts.
\end{abstract}

\section{Introduction}

Tissue-engineered articular cartilage has been a subject of research for a number of years (eg. [1, 2]). Although difficulties with cartilage structure and integration still persist, techniques using combined grafts using cancellous bone and autologous cartilage are approaching clinical application ([3], [4, [5]). Common to all therapies with in vitro engineered autografts is the need for optimal fit of the implant, which is imperative for screwless implantation and successful integration. Computer Assisted Surgery (CAS) techniques as used in orthopaedic surgery (e.g. [6]) have the potential both to ensure the required accuracy and simplify the therapy. A pilot study was hence conducted aiming at assembling a set of methods to realise and prove the feasibility of computer assisted arthroplasty using bioengineered autografts. The ankle joint was chosen as a first target 
because of the lack of suitable alternatives: post-traumatic osteoarthritis can be diagnosed in patients as young as 20 years, where classic therapies like total ankle joint arthroplasty using an artificial prosthesis or arthrodesis with fixation screws have considerable drawbacks (loss of mobility, poor long-term outcome expectation, difficult revision).

At the Universitätsklinikum Freiburg, Germany, one case of post - traumatic osteoarthritis has been treated with a bioengineered implant. The intervention was conducted in two steps: one for arthrotomy and defect moulding, a second for implanting the bio-engineered construct. Between the two operations several weeks were needed to proliferate autologous chondrocytes and let them integrate into a cancellous bone construct shaped after the defect mould. Albeit clinically successful, this procedure does not lend itself well to routine application: the two-step operation, the long period of treatment and the high cost of individually constructed autografts make it a time-consuming and costly alternative to classical therapies. Using CAS technologies, the procedure can be significantly simplified and generalised to allow pre-fabrication of implant parts. The revised procedure consists of planning based on $\mathrm{CT}$ image data, harvesting mesenchymal stem cells by needle biopsy, constructing the autograft according to the planning and conducting one single intervention for the arthrotomy and construct implantation. The defect debridement has to be accurate enough to make the pre-constructed graft fit without a screw; proving this accuracy in the context of a complete cycle of treatment was a main goal of this initial study.

\section{Methods}

\subsection{Rotational Symmetric Ankle Joint Model}

Based on the hinge-like articulation of the upper ankle joint, a rotational symmetric joint was assumed in the region of interest for arthroplasty. This approach allows shape determination using a small number of points on the joint surface, effectively circumventing the need for CT segmentation. A new software was developed to define the ankle joint shape model interactively on this basis. It consists of two steps: determine the joint axis and define the rotational profile. To determine the joint axis, arbitrary joint surface points are identified on sagittal planes in the region of interest. On each plane the software performs a least-mean-square fit of a circle to the points, storing the circle center as one point on the rotation axis. A second least-mean-square fit is performed in 3D to find the optimal fit of a line through all circle centers, yielding the joint axis. The accuracy of this axis calculation depends on the number of points selected and on the anatomy of the individual joint. Selecting 40 to 80 surface points in a rotational symmetric region of Talus and/or Tibia usually gives good results; in this study, about 100 points were used per axis calculation.

Once the joint axis is established, a model for the joint follows directly from rotating a joint profile around the axis. Accordingly, the software allows interactive checking whether the target bone is sufficiently rotational symmetric in the area of interest. 


\subsection{Preoperative Planning and Construct Manufacturing}

The axis determination software was further extended to allow the interactive planning of ankle joint arthroplasty prior to the operation. The planning consists of four steps:

1. Determine the joint axis

As described in chapter 2.1, arbitrary points are selected on sagittal planes in the region of interest. The software performs an optimal fit of a rotational symmetric body to the points selected to determine the joint axis.

2. Determine the lateral graft profile

A "hub view" is defined along the joint axis. In this view, a "U" profile can be defined determining the front, bottom and rear face of the implant (Fig. 13). The hub view can be shifted along the joint axis to determine the optimal section of the joint to be replaced.

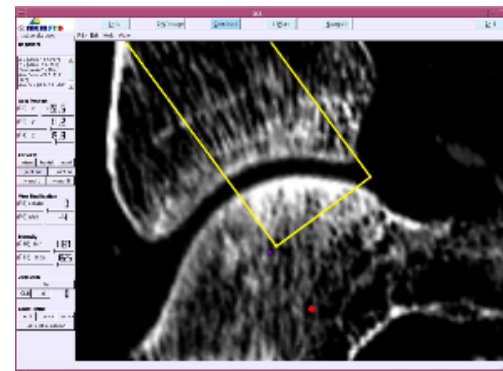

a) lateral view

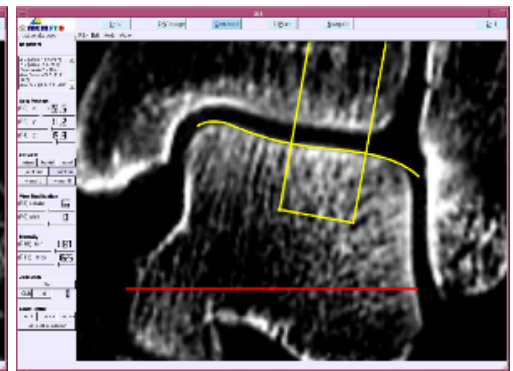

b) frontal view

Fig. 1. Determine the implant profiles in the two characteristic views

3. Determine the frontal graft profile

A "cut view" is defined through the joint axis. The axis is always shown horizontally, with the current view rotating around it. In this view, a profile can interactively be defined consisting of an "U" shape and a spline-interpolated curve following the joint surface (Fig. 1b). A landmark is set at an arbitrary position to localise the $\mathrm{U}$ shape in $3 \mathrm{D}$.

4. Visualize the resulting construct shape

Having defined the implant shape from hub view and from cut view, the visualisation is done using the CAD software SolidWorks@) (SolidWorks Corporation, Concord, U.S.A.). The output of the shape is stored in a CAM environment (construct manufacturing) as well as in our institute's CAS environment.

The CAD part description of the planned implant shape was used to program a CAM device to manufacture the dummy implant. The implants were custom milled according this plan in PU plastic "ep-Dur" (Emaform AG, Gontenschwil, Switzerland). 


\subsection{Preparing the CAS Environment}

At our institute a modular CAS platform has been developed allowing efficient and re-usable implementation of applications for computer assisted surgery. This study was conducted using an active Optotrak $₫$ high resolution optical tracking system from Northern Digital Inc., Waterloo, Canada. Tests have been carried out with angled chisels to study their behaviour in human bone and the feasibility limits of possible construct shapes. For navigated ankle joint arthroplasty, a set of three chisels has been designed and tested: one chisel with a blade angled $90^{\circ}$, one with a straight blade, $10 \mathrm{~mm}$ tip bent by $45^{\circ}$ and one chisel with a straight blade, $9.5 \mathrm{~mm}$ tip. Experiments on a human cadaver showed that the chisels tested are well suited for generating the planned defects. Infrared markers were attached to the bone under treatment and to every surgical tool used. In-house image processing algorithms were used to establish the correspondence between bone, tools and the visualised CT image by pair-point matching of landmarks on the bone. The result was improved by matching arbitrary surface points. The result of the preoperative planning, the implant shape and position, was imported into the CAS system. The contours of the implant were visualised in the CT image to allow navigated operation according to the plan. Surgical tools were tracked by the Optotrak® Camera and visualised at their proper position in the CT volume. Instead of showing the actual physical appearance of the chisels, aiming models were displayed as shown in Fig. 2 a, allowing a precise navigation of the tools (Fig. 2b).

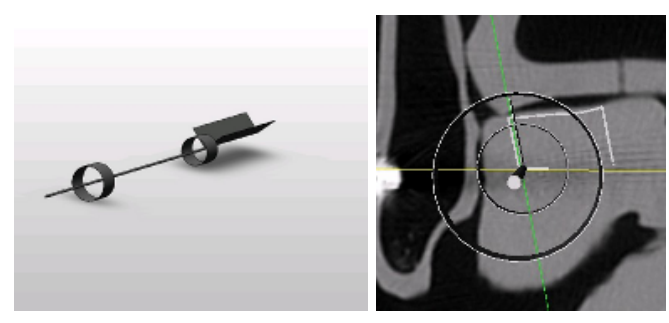

a) Aiming guide

b) Navigation screen

Fig. 2. Navigated Arthrotomy

\subsection{Pre-trial Using a Plastic Model and First Accuracy Evaluation}

A first trial combining the main elements of the target therapy was made using a plastic foot model from 3B Scientific GmbH, Hamburg, Germany. One talar implant shape was planned based on a preoperative CT. The operation was conducted using the chisels and analysed with postoperative CT. For this preliminary trial, no dummy implant was manufactured. The defect was analysed comparing distances on pre-op and post-op CT images (see Fig. 33) and double-checking them with measures on the plastic model. The error measures are shown in Fig. 3b and listed in Table 1 . 


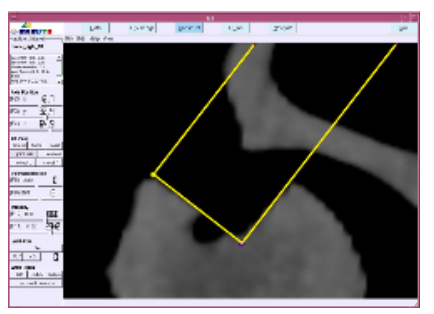

a) Postoperative CT, hub view b) Dimensions measured

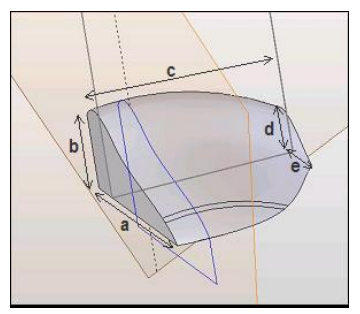

Fig. 3. Postoperative analysis of the pre-trial

Table 1. Postoperative measurements of the pre-trial operation

\begin{tabular}{|l|c|c|c|c|}
\multicolumn{2}{|l|}{ Distance } & $\begin{array}{c}\text { Planned Value } \\
{[\mathrm{mm}]}\end{array}$ & $\begin{array}{c}\text { Achieved Value } \\
{[\mathrm{mm}]}\end{array}$ & $\begin{array}{l}\text { Error } \\
{[\mathrm{mm}]}\end{array}$ \\
\hline a & medial depth & 14.58 & 14.10 & 0.48 \\
\hline b & medial height & 8.87 & 8.40 & 0.47 \\
\hline c & rear width & 15.45 & 15.20 & 0.35 \\
\hline d & lateral height & 11.02 & 9.50 & 1.52 \\
\hline e & lateral depth & 17.17 & 14.10 & 3.07
\end{tabular}

This analysis revealed a software error displaying the implant shape which affected the lateral face of the planned defect. Measurement of the other distances showed results well below $1 \mathrm{~mm}$.

\section{Results}

A trial operation was conducted on one cadaveric human foot. For the first time a closed cycle of treatment was simulated from pre-operative planning to implanting a custom graft shaped using the rotational symmetric model.

A first intuitive result is how well the implant fits into the defect made. In the experiment, proper fixation of the graft was ensured by driving it into the defect using a hammer and pestle. Visual inspection showed a good restoration of the joint surface, with some locations where the surrounding bone had broken off due to the chiseling. Live bone, being less brittle as cadaveric one, can be expected to allow generally better results.

Second, the defect was measured manually in the post-operative CT image; the results are shown in Fig. 4a, b (dorsal view) and Fig. 5a, b (lateral view). The "U" shape of the graft profile is displayed on the post-operative image exactly as planned preoperatively. Table 2 gives an overview over the dimensions as observed in the postoperative image.

The outcome was further analysed by segmenting both pre- and post-operative images, performing a normalized mutual information registration ([7]) and calculating the difference to obtain a surface model of the defect. A graphical view of 


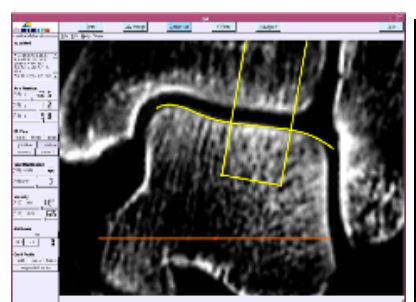

a) Planned profile

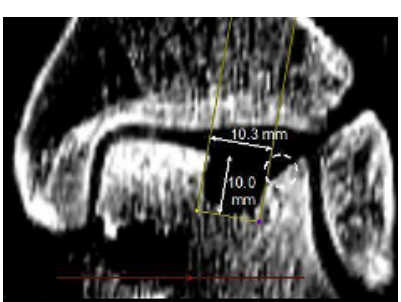

b) post-operative $\mathrm{CT}$

Fig. 4. Dorsal view of the operative result

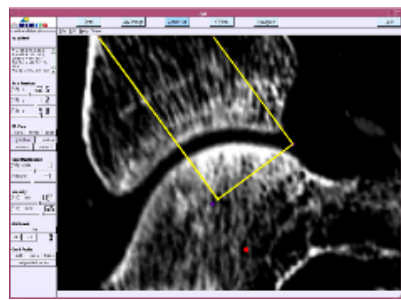

a) Planned profile

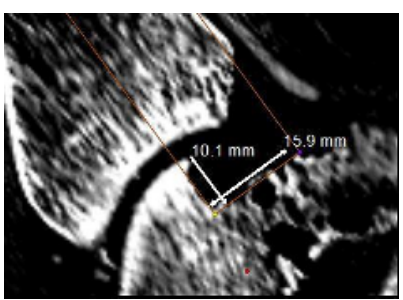

b) post-operative $\mathrm{CT}$

Fig. 5. Lateral view of the operative result

Table 2. Accuracy measurement using the post-operative CT image

\begin{tabular}{|l|c|c|c|c|}
\multicolumn{2}{|l|}{ Distance } & $\begin{array}{c}\text { Planned Value } \\
{[\mathrm{mm}]}\end{array}$ & $\begin{array}{c}\text { Achieved Value } \\
{[\mathrm{mm}]}\end{array}$ & $\begin{array}{c}\text { Error } \\
{[\mathrm{mm}]}\end{array}$ \\
\hline $\mathrm{a}$ & medial depth & 15.80 & 15.1 & 0.7 \\
\hline $\mathrm{b}$ & medial height & 9.64 & 9.5 & 0.1 \\
\hline c & rear width & 10.27 & 9.3 & 1.0 \\
\hline $\mathrm{d}$ & lateral height & 9.11 & 10.3 & 1.2 \\
\hline e & lateral depth & 15.26 & 16.4 & 1.1
\end{tabular}

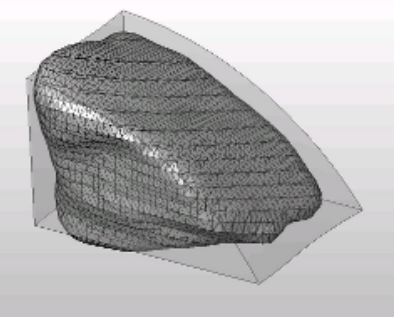

a) $3 \mathrm{D}$ view

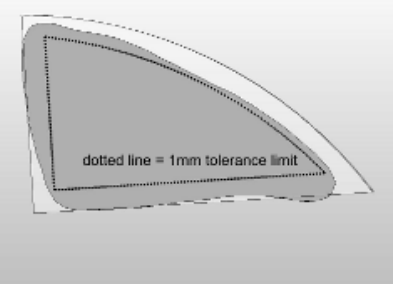

b) lateral view with $1 \mathrm{~mm}$ tolerance

Fig. 6. Analysis of the segmented defect 


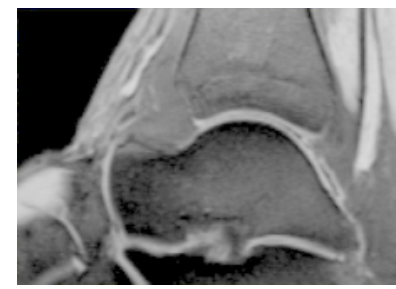

a) Preoperative MRI
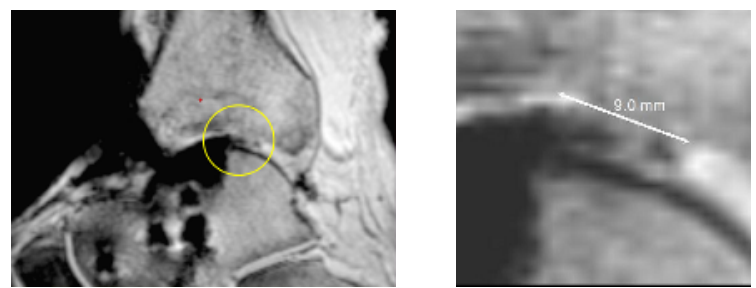

b) post-op MRI; damage encircled c) tibial cartilage damage

Fig. 7. Medial view of the operative result in MRI

this analysis is shown in Fig. 6a. The lateral profile (Fig. 6b) shows the defect surface to be well within an error limit of $1 \mathrm{~mm}$, which can be considered clinically successful.

A last analysis was conducted using post-operative MRI imaging (Fig. 7). The dummy autograft, manufactured in PU plastic, is not visible. While showing intact cartilage on the Talus side, the Tibia side has been damaged during the operation; for clinical application, the tools used need to be further optimised to avoid these injuries.

\section{Discussion and Conclusion}

The presented study does successfully prove that CAS techniques can be applied to support ankle joint arthroplasty using bio-engineered autografts. It is possible to make a custom-built bio-implant in a parameterisable shape planned on the basis of CT images and to implant it successfully at the planned site.

The proof of this hypothesis was done assuming a rotational symmetric shape model of the ankle joint surface, which is only correct for a limited part of talus and tibia. A more general model (e.g. 8]) could improve the matching of implant surface and surrounding joint surface, while at the same time making the technique applicable to other joints with a different kind of articulation. A big advantage of the rotational symmetric model is that no preoperative segmentation of the CT volume is required - a task which is difficult to achieve automatically, especially in joints with a narrow cavity. Manual segmentation is a very time-consuming alternative and would make the method impractical for clinical application. The determination of the ankle joint axis from the joint surface as developed in this project could be an interesting base for further studies: for example, the axis derived from the tibial or talar surface could be compared with each other and used for diagnosis. For that purpose, a closer investigation of its accuracy is required, which was not part of this study. By computing a segmented profile of the difference between pre- and post-operative images, the study tried to extract objective measures for surface errors of the resulting defect. While being sufficient to show the general feasibility, further work is needed to objectively analyse a series of interventions. This is particularly desirable for the actual joint surface, which is the main clinically relevant parameter, provided 
the implant is properly fixed in the bone. Such a surface quality measure could also be helpful to optimise the conditions for graft integration.

\section{Acknowledgements}

This study was funded by the AO Foundation, Davos, Switzerland. We would like to thank Mrs. E. Spielvogel and Dr. M. Bonél of Insel Hospital, Bern, Switzerland, for providing CT and MRI images and for their radiological expertise; Dr. J. Kowal et al for building MEM center's CAS platform used to perform the operation; U. Rohrer and T. Gerber for manufacturing implants and operating tools.

\section{References}

1. Caplan, A.e.a.: Principles of cartilage repair and regeneration. Clinical Orthopaedics and Related Research (1997) 254-269

2. Klemt, C.: Tissue engineered cartilage with cultured chondrocytes and a collagen sponge matrix. In G.B., S., Horch, R., Tanczos, E., eds.: Biological Matrices and Tissue Reconstruction. Number 1682 in LNCS, Springer (1998) 169-172

3. Oakes, B.: Orthopaedic tissue engineering: from laboratory to the clinic. Medical Journal of Australia 180 (2004) S35-S38

4. Lynn, A., Brooks, R., Bonfield, W., Rushton, N.: Repair of defects in articular joints. Journal of Bone and Joint Surgery (Br) 86 (2004) 1093-9

5. Landis, W.e.a.: The potential of tissue engineering in orthopedics. Orthopedic Clinics of North America 36 (2005) 97-104

6. Nolte, L., Ganz, R., eds.: Computer Assisted Orthopedic Surgery (CAOS). Hogrefe and Huber (1999)

7. Studholme, C., Hill, D., Hawkes, D.: An overlap invariant entropy measure of 3d medical image alignment. Pattern Recognition 32 (1999) 71-86

8. Gerig, G., Styner, M., Szekely, G.: Statistical shape models for segmentation and structural analysis. IEEE International Symposium on Biomedical Imaging (2002) 